University of Wollongong

Research Online

Faculty of Engineering and Information

Faculty of Engineering and Information

Sciences - Papers: Part A

Sciences

$1-1-2014$

Multi-stage compressed sensing and wall clutter mitigation for through-thewall radar image formation

Fok Hing Chi Tivive

University of Wollongong, tivive@uow.edu.au

Abdesselam Bouzerdoum

University of Wollongong, bouzer@uow.edu.au

Van Ha Tang

University of Wollongong, vht986@uowmail.edu.au

Follow this and additional works at: https://ro.uow.edu.au/eispapers

Part of the Engineering Commons, and the Science and Technology Studies Commons

Research Online is the open access institutional repository for the University of Wollongong. For further information contact the UOW Library: research-pubs@uow.edu.au 


\title{
Multi-stage compressed sensing and wall clutter mitigation for through-the-wall radar image formation
}

\begin{abstract}
In this paper, a multi-stage through - The-wall radar imaging technique combining wall clutter mitigation and scene reconstruction is proposed. In the first stage, compressed sensing is applied to compressive measurements to recover the radar signals in the wavelet domain. Then, a subspace projection method is employed to remove the wavelet coefficients associated with the exterior wall reflections. In the second stage, the remaining wavelet coefficients are further compressed using principal component analysis. A compact linear measurement model is then formulated which relates the compressed wavelet coefficients to the image of the scene. Finally, the image reconstruction problem is solved in a more efficient compressed sensing framework, using the compact linear measurement model. Experiment results obtained from real data prove that the proposed method is more efficient and achieves better performance, in terms of target-to-clutter ratio, than direct compressed sensing signal recovery method and delay-and-sum beamforming. 2014 IEEE.
\end{abstract}

\section{Keywords}

radar, mitigation, clutter, image, formation, sensing, wall, multi, stage, compressed

Disciplines

Engineering | Science and Technology Studies

\section{Publication Details}

F. Hing Chi. Tivive, A. Bouzerdoum \& V. Ha. Tang, "Multi-stage compressed sensing and wall clutter mitigation for through-the-wall radar image formation," in 2014 IEEE 8th Sensor Array and Multichannel Signal Processing Workshop (SAM), 2014, pp. 489-492. 


\title{
Multi-Stage Compressed Sensing and Wall Clutter Mitigation for Through-the-Wall Radar Image Formation
}

\author{
Fok Hing Chi Tivive, Abdesselam Bouzerdoum, and Van Ha Tang \\ School of Electrical, Computer and Telecommunications Engineering \\ University of Wollongong, Wollongong, NSW 2522, Australia \\ Email: $\{$ tivive, bouzer, and vht986\}@uow.edu.au
}

\begin{abstract}
In this paper, a multi-stage through-the-wall radar imaging technique combining wall clutter mitigation and scene reconstruction is proposed. In the first stage, compressed sensing is applied to compressive measurements to recover the radar signals in the wavelet domain. Then, a subspace projection method is employed to remove the wavelet coefficients associated with the exterior wall reflections. In the second stage, the remaining wavelet coefficients are further compressed using principal component analysis. A compact linear measurement model is then formulated which relates the compressed wavelet coefficients to the image of the scene. Finally, the image reconstruction problem is solved in a more efficient compressed sensing framework, using the compact linear measurement model. Experiment results obtained from real data prove that the proposed method is more efficient and achieves better performance, in terms of targetto-clutter ratio, than direct compressed sensing signal recovery method and delay-and-sum beamforming.
\end{abstract}

\section{INTRODUCTION}

In remote sensing applications, through-the-wall radar imaging (TWRI) is becoming a viable technology for imaging enclosed building structures. It can be used for various purposes, including determining the building layout, and locating and identifying stationary objects behind walls [1]. In TWRI, high resolution is obtained by transmitting ultra-wideband signals using a large aperture array; this results in prolonged data acquisition and processing time. Thus, the research focus in TWRI has shifted towards scene reconstruction under reduced measurements, in order to address certain logistical constraints and provide prompt situation awareness in an efficient and reliable way.

Compressed sensing studies have shown that sparse and compressible signals can be accurately recovered from far fewer nonadaptive linear measurements than required by the Nyquist-Shannon sampling theorem [2], [3]. A number of techniques based on compressed sensing (CS) have been developed for efficient image reconstruction from reduced measurements [4]-[9]. The scene reconstruction is posed as an inverse problem, where a linear model is employed to link the selected measurements directly to the imaged scene, which is assumed to be sparse. Recently, a CS-based method has been proposed which combines wall clutter mitigation and image formation [10]. Wall clutter mitigation is performed on the space-frequency measurements using spatial filtering [11] or a subspace projection method [12]. The range profiles, which are obtained from the reduced measurements, are transformed back to the signal domain, and the image is formed using the same set of frequencies used to collect the initial measurements.

In this paper, a two-stage image formation method is developed for TWRI. The rationale for a two-stage approach is to first recover the radar signal accurately in order to employ wall clutter mitigation before image formation. In the second stage, principal component analysis (PCA) is employed to compress the estimated sparse coefficients and remove noise; thereby, a compact linear model, which retains most of the target information, is established for fast and more accurate scene reconstruction.

The remainder of the paper is organized as follows. Section II presents the basic CS-based image formation model for TWRI. Section III describes the wall clutter mitigation and image formation method using compressed sensing. Section IV discusses the experimental results and comparisons based on real data. Section V presents concluding remarks.

\section{CS IMAGE FORMATION MODEL FOR TWRI}

Consider a mono-static stepped-frequency TWRI system for high-resolution imaging. A transceiver is placed at several scan positions parallel to the wall to synthesize an $N$-element line array. The scene is interrogated with a wideband signal by transmitting a stepped-frequency signal of $M$ frequencies, equispaced over the desired bandwidth $\omega_{M-1}-\omega_{0}$,

$$
\omega_{m}=\omega_{0}+m \Delta \omega, \quad m=0,1, \ldots, M-1
$$

where $\omega_{0}$ is the lowest frequency in the desired frequency band and $\Delta \omega$ is the frequency step size. Without loss of generality, we assume the wall is homogeneous and behaves as a specular reflector, and the scene behind the wall contains $P$ point-like target of constant reflectivity $\sigma_{\mathrm{t}}$, independent of frequency and aspect angle. The radar signal associated with the $m$-th frequency received by the $n$-th antenna is given by

$$
y(m, n)=\sigma_{\mathrm{w}} \exp \left(-j \omega_{m} \tau_{n, \mathrm{w}}\right)+\sum_{p=1}^{P} \sigma_{p} \exp \left(-j \omega_{m} \tau_{n, p}\right),
$$

where $\sigma_{\mathrm{w}}$ is the wall complex reflectivity, and $\tau_{n, p}$ and $\tau_{n, \mathrm{w}}$ are the two-way wave propagation delays from the $n$-th antenna to the $p$-th target and the wall, respectively. The imaged scene 
is divided into a rectangular grid consisting of $Q$ pixels. Let $\boldsymbol{y}_{n}=[y(0, n), \ldots, y(M-1, n)]^{T}$ denote the vector containing the measurements collected at the $n$-th antenna location and $s_{i}$ denote a weighted indicator function, defined as

$$
s_{i}= \begin{cases}\sigma_{p}, & \text { if the } p \text {-th target occupies the } i \text {-th pixel } \\ \sigma_{\mathrm{w}}, & \text { if the wall occupies the } i \text {-th pixel; } \\ 0, & \text { otherwise. }\end{cases}
$$

Using the above indicator function, we can form a column vector $s=\left[s_{1}, \ldots, s_{Q}\right]^{T}$ representing the imaged scene. Let $\tau_{n, q}$ denote the focusing delay between the $n$-th antenna and the $q$-th pixel. The radar signal received by the $n$-th antenna can be written in matrix-vector form as

$$
\boldsymbol{y}_{n}=\Psi_{n} \boldsymbol{s}
$$

where $\Psi_{n}=\left[\psi_{n}(m, q)\right]$ is an $M \times Q$ matrix with the $m q$-th element given by $\psi_{n}(m, q)=\exp \left(-j \omega_{m} \tau_{n, q}\right)$. Now, the relation between the measurements collected across the array aperture and the scene $s$ can be expressed as

$$
\boldsymbol{y}=\Psi s,
$$

where $\boldsymbol{y}=\left[\boldsymbol{y}_{0}^{T}, \boldsymbol{y}_{1}^{T}, \ldots, \boldsymbol{y}_{N-1}^{T}\right]^{T}$ and $\Psi=\left[\Psi_{0}^{T}, \Psi_{1}^{T}, \ldots, \Psi_{N-1}^{T}\right]^{T}$.

According to compressed sensing theory, a sparse scene $s$ can be successfully recovered from a reduced set of $K$ measurements $(K \ll M N)$, which can be obtained by applying a random selection matrix $\Phi \in \mathbb{R}^{K \times M N}$. Thus, the compressed measurement model can be expressed as

$$
z=\Phi y=\Phi \Psi s
$$

From the compressed measurement vector $z$, a sparse signal $s$ can be obtained by solving the following optimization problem:

$$
\min \|s\|_{1} \quad \text { subject to }\|z-\Phi \Psi s\|_{2} \leq \delta
$$

where $\|s\|_{1}$ denotes the 1 -norm of $s$ and $\delta$ is a noise bound. Equation (7) can be solved using a CS sparse recovery algorithm and the solution is considered as the image of the scene. However, a wall clutter mitigation technique needs to be applied before image formation in order to reveal the targets of interest residing behind the wall.

\section{Proposed CS-BASEd Image Formation Method}

The proposed method combines wall clutter mitigation and image formation. The first stage of the proposed method is to recover the radar signal accurately using a wavelet dictionary and attenuate the wall clutter, by removing the signal components associated with the front wall returns. In the second stage, a compact linear model is established which relates the remaining signal components to the image of the scene. Finally, the image is reconstructed by solving another CS problem.

\section{A. Radar Signal Recovery}

At each antenna location, a subset of frequencies is selected to reconstruct the radar signal. Here, sparse representation using a wavelet dictionary is employed to recover the full measurement vector. Given a set of $M$ orthonormal wavelet basis functions $\mathbf{W} \in \mathbb{R}^{M \times M}$, the signal $\boldsymbol{y}_{n}$ of Eq. (4) can be expressed as

$$
\boldsymbol{y}_{n}=\mathbf{W} \boldsymbol{x}_{n}
$$

where $\boldsymbol{x}_{n}$ is a sparse vector of wavelet coefficients. Using a selection matrix $\phi_{n}$ to choose a subset of frequencies, we can express the reduced measurement vector as

$$
\boldsymbol{z}_{n}=\phi_{n} \boldsymbol{y}_{n}=\phi_{n} \mathbf{W} \boldsymbol{x}_{n}
$$

From the reduced measurement vector $z_{n}$, the wavelet coefficient vector can be recovered by solving the following problem:

$$
\min \left\|\boldsymbol{x}_{n}\right\|_{1} \quad \text { subject to }\left\|\boldsymbol{z}_{n}-\phi_{n} \mathbf{W} \boldsymbol{x}_{n}\right\|_{2} \leq \delta
$$

The next step is to remove the wavelet coefficients associated with the wall returns.

\section{B. Wall Clutter Mitigation}

After the signal recovery, different wall clutter mitigation methods can be used. Here, a subspace projection method is applied to remove the wavelet coefficients associated with the wall returns [12], [13]. Let $\mathbf{X}$ denote the matrix containing the wavelet coefficients recovered from all antenna positions, $\mathbf{X}=\left[\boldsymbol{x}_{0}, \boldsymbol{x}_{1}, \ldots, \boldsymbol{x}_{N-1}\right]$. Using SVD, matrix $\mathbf{X}$ can be decomposed into a set of singular vectors, $\mathbf{U}=\left[\boldsymbol{u}_{1}, \ldots, \boldsymbol{u}_{L}\right]$ and $\mathbf{V}=\left[\boldsymbol{v}_{1}, \ldots, \boldsymbol{v}_{N}\right]$, and their corresponding singular values. The wall subspace can be defined as

$$
\mathbf{P}_{\mathrm{w}}=\sum_{i \in \mathcal{D}} \boldsymbol{u}_{i} \boldsymbol{v}_{i}^{H}
$$

where $\mathcal{D}$ denotes the index set of the singular vectors spanning the wall subspace. These singular vectors are determined by applying a threshold technique to the singular value spectrum [13]. To remove the wavelet coefficients associated with the wall returns, the matrix $X$ is projected onto the orthogonal subspace to the wall subspace

$$
\widetilde{\mathbf{X}}=\left(\mathbf{I}-\mathbf{P}_{\mathrm{w}} \mathbf{P}_{\mathrm{w}}^{H}\right) \mathbf{X},
$$

where $\mathbf{I}$ is the identity matrix. After the projection operation, the wavelet coefficients may still contain some wall residuals. Thus, we transform the wavelet coefficients into radar signals and compute their corresponding range profiles by applying the inverse fast Fourier transform. Before the transformation, we apply a window function to the recovered signal to reduce sidelobes. Based on the range profile, we employ a segmentation technique described in [13] to further mitigate wall residual. The processed range profiles are transformed back to form wavelet coefficients associated with the target signal, $\widetilde{\boldsymbol{x}}_{n}, n=0, \ldots, N-1$. 


\section{Scene Reconstruction}

From Eq. (8), we can obtain the full measurement vector $\boldsymbol{y}_{n}$ at each antenna location, then apply a backprojection method for image formation, similar to the approach described in [7]. Alternatively, we can solve the minimization problem given in Eq. (7) using the measurements computed from the processed wavelet coefficients. However, here we first develop a compact linear model which relates the wavelet coefficients directly to the imaged scene. Using Eqs. (8) and (4), we can express the wavelet coefficients $\widetilde{\boldsymbol{x}}_{n}$ as

$$
\widetilde{\boldsymbol{x}}_{n}=\mathbf{W}^{T} \psi_{n} \widetilde{\boldsymbol{s}} .
$$

where $\mathbf{W}^{T}$ represents the inverse wavelet transform since $\mathbf{W}$ is an orthogonal matrix and $\widetilde{\boldsymbol{s}}$ denotes the formed target image free of wall clutter. The new measurement vectors, $\widetilde{\boldsymbol{x}}_{n}$, obtained from all antenna locations can be concatenated to form a composite measurement vector, which can be used to reconstruct the image of the scene. However, this approach yields a very high-dimensional measurement vector (of size $N M \times 1$ ) and a huge dictionary (of size $N M \times Q$ ), which leads to inefficient image reconstruction. Let $\mathbf{C}$ denote the covariance matrix of the new measurements given in (13). Using the eigendecomposition, we can write $\mathbf{C}=\mathbf{G} \Lambda \mathbf{G}^{H}$, where $\mathbf{G}=\left[\boldsymbol{g}_{1}, \ldots, \boldsymbol{g}_{L}\right]$ is a matrix of eigenvectors and $\Lambda$ is a diagonal matrix of eigenvalues, arranged in descending order. We define a projection matrix $\mathbf{P}=\left[\boldsymbol{g}_{1}, \ldots, \boldsymbol{g}_{K}\right]$ consisting of the first $K(K \ll M)$ eigenvectors; $K$ is determined using an information theoretic criterion, such as Akaike Information Criterion (AIC) or Minimum Description Length (MDL) [14]. Applying the projection matrix $\mathbf{P}^{H}$ to (13) yields the compressed measurement vector $\widehat{\boldsymbol{x}}_{n}=\mathbf{P}^{H} \widetilde{\boldsymbol{x}}_{n} \in C^{K}$,

$$
\widehat{\boldsymbol{x}}_{n}=\mathbf{P}^{H} W^{\dagger} \psi_{n} s=\mathbf{D}_{n} \widetilde{\boldsymbol{s}} .
$$

Now, concatenating all compressed measurement vectors into a composite vector $\widehat{\mathbf{x}}=\left[\widehat{\boldsymbol{x}}_{1}^{T}, \cdots, \widehat{\boldsymbol{x}}_{N}^{T}\right]^{T}$ and arranging the individual compressed dictionaries into a composite dictionary $\mathbf{D}=\left[\mathbf{D}_{1}^{T}, \cdots, \mathbf{D}_{N}^{T}\right]^{T}$, we obtain the following compact linear model

$$
\widehat{\mathbf{x}}=\mathbf{D} \widetilde{\boldsymbol{s}},
$$

where $\widehat{\mathbf{x}}$ is $N K \times 1$ and $\mathbf{D}$ is $N K \times Q$. For image formation, we solve the following minimization problem:

$$
\min \|\widetilde{\boldsymbol{s}}\|_{1} \quad \text { subject to }\|\widehat{\mathbf{x}}-\mathbf{D} \widetilde{\boldsymbol{s}}\|_{2} \leq \delta .
$$

After using a CS recovery algorithm to solve (16), we take the magnitude of $s$ as the image of the scene free of wall clutter.

\section{EXPERIMENTAL RESULTS}

Real radar data are collected in the Radar Imaging Lab of the Center for Advanced Communications at Villanova University. The TWRI scene consists of nine targets: three dihedrals, four trihedrals, a sphere, and a tophat. Each target is placed at a certain distance from the wall. The wall between the radar and the targets is a drywall of thickness $0.127 \mathrm{~m}$, built from a wooden frame with one side fastened with $0.019 \mathrm{~m}$ plywood and the other side with $0.0158 \mathrm{~m}$ gypsum wall board.
A stepped-frequency radar of $1 \mathrm{GHz}$ bandwidth centered at $2.5 \mathrm{GHz}$ with a frequency step size of $5 \mathrm{MHz}$ is used to image the targets behind the wall. The transceiver is placed at 69 different positions to synthesize an array aperture of length $1.2246 \mathrm{~m}$. The interested reader is referred to [15] for a detailed description of the experimental setup.

A set of 13,869 space-frequency measurements, i.e., (201 frequencies $\times 69$ antennas) are collected using the TWRI system. In practice, not all radar signals from all antennas across the array aperture can be acquired successfully due to occlusions by metallic obstacles. Thus, a set of 41 antennas and 121 frequencies are randomly selected for the experiments; in other words, only $35.77 \%$ of the total measurements are used for scene reconstruction.

In the first experiment, we compare the performance of the wavelet and Fourier dictionary when recovering the radar signal. The wavelet dictionary $\mathbf{W}$ is obtained using Daubechies 8 wavelet at two decomposition level. The recovered measurement vector at each antenna location is compared to the full measurement vector, available from the recorded experimental data. Figure 1 shows the normalized mean square error (NMSE) as a function of the antenna position. The recovered signals using a wavelet dictionary are clearly more accurate than those obtained with the Fourier basis; that is, they have a much lower NMSE. On average, the wavelet basis achieves an NMSE of 0.0146, whereas the Fourier basis produces an NMSE of 0.0295 .

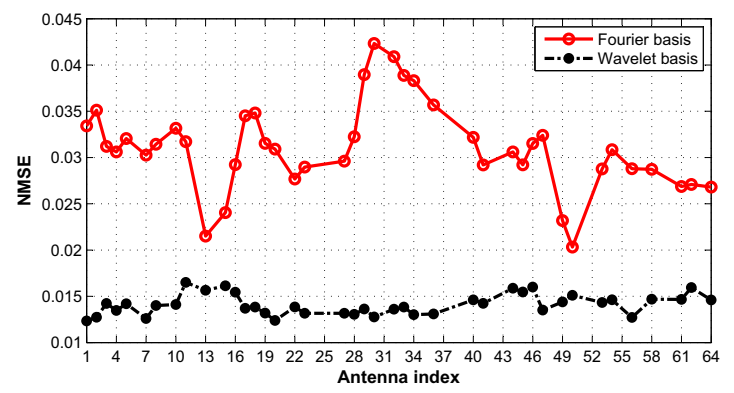

Fig. 1. Normalized mean square error of reconstructing the radar signal from the reduced set of space-frequency measurements when using Fourier and wavelet basis.

For comparison purposes, delay-and-sum (DS) beamforming, direct CS signal recovery, and the proposed method are employed for image formation using the same reduced measurement set. To quantify the quality of the formed images, the target-to-clutter ratio (TCR) is computed for each formed image

$$
\mathrm{TCR}=\frac{1}{N_{\mathrm{t}}} \sum_{q \in A_{\mathrm{t}}}|I(q)|^{2} / \frac{1}{N_{\mathrm{c}}} \sum_{q \in A_{\mathrm{c}}}|I(q)|^{2},
$$

where $|I(q)|^{2}$ is the squared magnitude of the $q$-th complexvalued pixel, $A_{\mathrm{t}}$ is the target region, $A_{\mathrm{c}}$ is the clutter region, $N_{\mathrm{c}}$ and $N_{\mathrm{t}}$ are, respectively, the number of pixels in the clutter region and target region; the clutter region is considered to be the entire image, excluding the target region.

Figure 2 presents the images obtained with the different image formation approaches. Figure 2(a) illustrates the image 


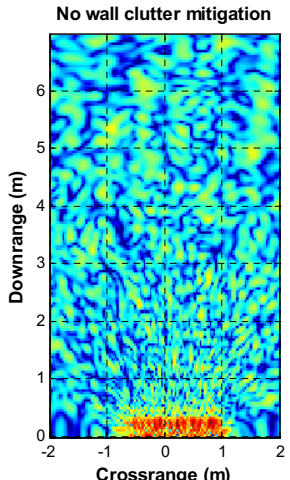

(a)

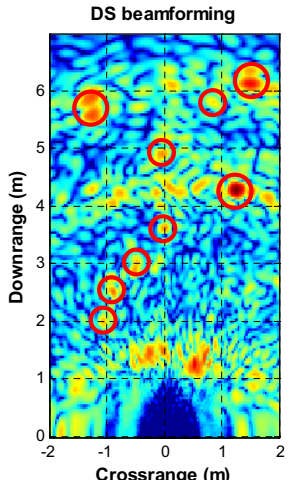

(b)

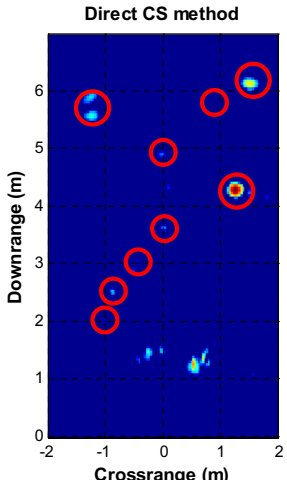

(c)

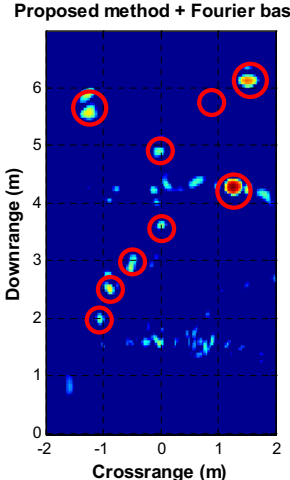

(d)

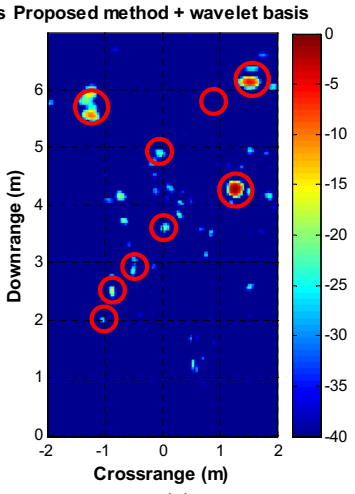

(e)

Fig. 2. With $60 \%$ of the total measurements, the image formed by different image formation method: (a) DS beamforming without wall clutter mitigation, (b) DS beamforming with wall clutter mitigation, (c) direct CS signal recovery method, (d) proposed method with Fourier basis and (e) proposed method with wavelet basis. NESTA [16] was used as the sparse recovery method for scene reconstruction.

formed using DS beamforming without any wall clutter mitigation; clearly the wall returns dominate, and none of the targets is visible. Figure 2(b) depicts the beamformed image after using the SVD-based wall clutter mitigation method to remove the wall clutter. Figure 2(c) presents the image formed by the direct CS signal recovery method, which has less clutter. By contrast, the proposed method produces images with strong targets, see Fig. 2(d) and 2(e). Furthermore, the image formed using the wavelet dictionary, Fig. 2(e), has slightly less clutter than the one obtained with Fourier basis, Fig. 2(d). The TCR values of the formed images depicted in Fig. 2 are listed in Table I. The proposed method achieves the highest TCR of $28.93 \mathrm{~dB}$, followed by the direct CS-method with a TCR of $24.72 \mathrm{~dB}$. DS beamforming gives a TCR of $8.49 \mathrm{~dB}$.

TABLE I

TARGET TO CLUTTER RATIO OF THE IMAGE FORMATION METHODS.

\begin{tabular}{|l|c|}
\hline Image formation approach & TCR $(\mathrm{dB})$ \\
\hline Proposed method with wavelet basis & 28.93 \\
\hline Proposed method with Fourier basis & 26.46 \\
\hline Direct CS method [10] & 24.72 \\
\hline DS beamforming with wall clutter mitigation & 8.49 \\
\hline
\end{tabular}

\section{CONCLUSION}

A new image formation method was proposed for TWRI, which performs wall clutter mitigation and scene reconstruction from a reduced set of measurements. In the first stage, a sparse representation using a wavelet dictionary is employed for signal recovery, followed by wall clutter mitigation. The remaining wavelet coefficients are then compressed and a compact linear model is established relating the compressed measurements in the wavelet domain to the image of the scene. In the final step, the image of the scene is obtained by solving a CS problem. Experimental results showed that the proposed method can effectively form an image free from wall clutter, and achieves higher TCR than the direct CS signal recovery method and DS beamforming.

\section{ACKNOWLEDGEMENT}

The authors would like to thank Prof. Moeness Amin and Dr. Fauzia Ahmad from the Center of Advanced Communications at
Villanova University, Villanova, PA, USA, for providing the experimental data.

\section{REFERENCES}

[1] M. G. Amin (Ed.), Through-the-wall radar imaging. Boca Raton, FL: CRC Press, 2010.

[2] D. L. Donoho, "Compressed sensing," IEEE Trans. Information Theory, vol. 52, no. 4, pp. 1289-1306, 2006.

[3] E. J. Candes, J. Romberg, and T. Tao, "Stable signal recovery from incomplete and inaccurate measurements," Communications on Pure and Applied Mathematics, vol. 59, no. 8, pp. 1207-1223, 2006.

[4] Q. Huang, L. Qu, B. Wu, and G. Fang, "UWB through-wall imaging based on compressive sensing," IEEE Trans. Geoscience and Remote Sensing, vol. 48, no. 3, pp. 1408-1415, 2010.

[5] M. Leigsnering, C. Debes, and A. M. Zoubir, "Compressive sensing in through-the-wall radar imaging," in Proc. IEEE Int. Conf. Acoustics, Speech, and Signal Processing, pp. 4008-4011.

[6] Y.-S. Yoon and M. G. Amin, "Imaging of behind the wall targets using wideband beamforming with compressive sensing," in Proc. IEEE/SP Workshop on Statistical Signal Processing, pp. 93-96.

[7] Y.-S. Yoon and M. G. Amin, "Through-the-wall radar imaging using compressive sensing along temporal frequency domain," in IEEE Int. Conf. on Acoustics Speech and Signal Processing, pp. 2806-2809.

[8] M. G. Amin and F. Ahmad, "Compressive sensing for through-the-wall radar imaging," J. of Electronic Imaging, vol. 22, no. 3, pp. 1-20, 2013.

[9] V. H. Tang, A. Bouzerdoum, and S. L. Phung, "Two-stage through-thewall radar image formation using compressive sensing," J. of Electronic Imaging, vol. 22, no. 2, pp. 1-10, 2013.

[10] E. Lagunas, M. G. Amin, F. Ahmad, and M. Najar, "Joint wall mitigation and compressive sensing for indoor image reconstruction," IEEE Trans. on Geoscience and Remote Sensing, vol. 51, no. 2, pp. 891-906, 2013.

[11] Y.-S. Yoon and M. G. Amin, "Spatial filtering for wall-clutter mitigation in through-the-wall radar imaging," IEEE Trans. on Geoscience and Remote Sensing, vol. 47, no. 9, pp. 3192-3208, 2009.

[12] F. H. C. Tivive, M. G. Amin, and A. Bouzerdoum, "Wall clutter mitigation based on eigen-analysis in through-the-wall radar imaging," in Proc. Int. Conf. on Digital Signal Processing, pp. 1-8.

[13] F. H. C. Tivive and A. Bouzerdoum, "An improved SVD-based wall clutter mitigation method for through-the-wall radar imaging," in Proc. IEEE 14th Workshop on Signal Processing Advances in Wireless Communication, pp. 425-429.

[14] M. Wax and T. Kailath, "Detection of signals by information theoretic criteria," IEEE Trans. on Acoustics Speech Signal Processing, vol. 33, no. 2, pp. 387-392, 1985.

[15] R. Dilsavor, W. Ailes, P. Rush, F. Ahmad, W. Keichel, G. Titi, and M. Amin, "Experiments on wideband through the wall imaging," in Proc. of the SPIE, Vol 5808, Symposium on Defense and Security, Algorithms for Synthetic Aperture Radar Imagery XII Conference, pp. 196-209.

[16] S. Becker, J. Bobin, and E. J. Candes, "Nesta: A fast and accurate firstorder method for sparse recovery," SIAM J. Imaging Sciences, pp. 1-39, 2011. 